\title{
Può essere davvero pericoloso esporsi acutamente all'alta quota senza allenamento?
}

\author{
Enrico Donegani, Flavio Chiarottino, Roberto Clemente, Franco Movalli, \\ Giovanni Venturino, Claudio Nuti, Elio Protto*
}

\begin{abstract}
Monaldi Arch Chest Dis 2006; 66: 230-231.
* Commissione Medica L.P.V. - C.A.I.

Corresponding author: Dott. Enrico Donegani; S.C. di Cardiochirurgia; ASO Maggiore della Carità; C.so Mazzini 18 - 28100 Novara;E-mail address: donegani@hotmail.com
\end{abstract}

Gli effetti dell'esposizione acuta all'altitudine sul sistema cardiovascolare sono stati ampiamente studiati negli anni passati [1-2]. Nei soggetti normali alcuni investigatori hanno riscontrato modesti aumenti dei valori pressori [3], altri una lieve riduzione [4], altri ancora nessuna modificazione [5]. Due articoli hanno riportato la risposta della pressione arteriosa all'altitudine in soggetti ipertesi, dimostrando un modesto aumento dei valori sistolici [3] in uno studio e nessun cambiamento nell'altro [5]. Generalmente questi studi sono sempre condotti su piccoli gruppi di soggetti sportivi e allenati alla quota, in pratica su una popolazione selezionata. Poco o nulla si conosce invece di cosa succede dal punto di vista emodinamico in soggetti normali o ipertesi in trattamento farmacologico, non allenati e senza alcuna preparazione specifica, occasionai i frequentatori della montagna e quindi maggiormente esposti ai pericoli di un'esposizione acuta all'alta quota.

Abbiamo voluto dunque valutare le variazioni dei più comuni parametri emodinamici e funzionali (pressione arteriosa, frequenza cardiaca e saturazione di ossigeno) in un gruppo di persone le quali hanno subito un rapido cambio di quota di oltre $2000 \mathrm{~m}$. salendo con la funivia in circa 2 ore da Alagna Val Sesia (1192 m.s.l.m.) a Punta Indren (massiccio del Monte Rosa, 3260 m.s.l.m.), in occasione della festività della Madonna della Neve del 5 agosto 2003.

73 soggetti volontari $(68 \%$ uomini, età media 55 anni) dei quali 60 autodefinitisi "normali" (42 uomini e 18 donne, età media 49 anni) e 13 con ipertensione arteriosa nota, in trattamento medico (8 uomini 5 donne, età media 61 anni), sono stati sottoposti alla partenza ad Alagna ad un breve questionario (età, abitudini, malattie, ecc) ed in tutti è stata rilevata la frequenza cardiaca (FC), la pressione arteriosa (PA) e la saturazione di $\mathrm{O} 2(\mathrm{SpO} 2)$. All'arrivo in quota a Punta Indren è stata ripetuta la misurazione dei medesimi parametri ed è stato chiesto ai soggetti se fossero comparsi disturbi particolari.

In sintesi, in tutti i soggetti la $\mathrm{SpO} 2$ si è abbassata da un valore medio normale del $98 \%$ ad un valore medio piuttosto basso del $90 \%$, ma solo il $15 \%$ di essi ha riferito il disturbo della dispnea. La FC è aumentata in tutti i soggetti ma mediamente mai in modo significativo $(+10 \%)$. Una piccola percentuale
(12\%) ha riferito sensazione di cardiopalmo (FC compresa tra $90 / \mathrm{m}$ ' e $140 / \mathrm{m}$ ') mentre gli altri, anche con frequenze superiori a 100/m', non hanno segnalato tale disturbo. Il comportamento della PA riscontrato del nostro studio è quello descritto da altri $\mathrm{Au}$ tori [3]. Nel gruppo di soggetti normali, nell' $80 \%$ dei casi la PA è restata costante, mentre nell' $7 \%$ è aumentata con l'esposizione acuta all'altitudine (PA diastolica $+20 \%$ ). Nel restante $11 \%$ di casi, con un'età media di 49 anni, è stata riscontrata un'ipertensione arteriosa misconosciuta che ovviamente si è mantenuta tale anche in quota. Nel gruppo di soggetti ipertesi e ben controllati in condizioni basali(Bbloccanti, sartanici, ACE-1) si è registrato un lieve aumento della PA diastolica in quota, nonostante la terapia, peraltro non significativo $(+7 \%)$. I disturbi soggettivi riferiti dal $38 \%$ delle persone all'arrivo in quota a Punta Indren come cefalea, nausea, astenia, vertigini, non sono risultati correlati ad alcuna variazione dei parametri emodinamici valutati.

I nostri risultati sono sovrapponibili a quelli descritti in bibliografia in soggetti allenati [6] e a quelli rilevati in un ampio studio condotto nel 2000 dalla Commissione Medica Centrale del CAI su oltre 12000 iscritti al sodalizio, una popolazione alpinistica abituale con un buon grado di preparazione fisica e con una frequentazione media della montagna di 23 anni [7].

Si può concludere che il mancato acclimatamento all'altitudine è sicuramente responsabile della genesi dei sintomi del mal di montagna nei soggetti esposti acutamente all'alta quota ma che non esistono differenze di comportamento fra persone abituali e quelle occasionali e che non sembra necessario uno specifico allenamento almeno per trascorrere una tranquilla e piacevole giornata di festa in alta montagna.

\section{Bibliografia}

1. Lenfant C, Sullivan K. Adaptation to high altitude. N Engl J Med 1971; 284: 1298-1309.

2. Heath H, Reid Williams D. Man at High Altitude. The Pathophysiology of Acclimatization and Adaptation. Edinburgh, London, Melbourn, New York: Churchill Livingstone, 1981: 1-347. 
3. Palatini P, Businaro R, Berton G, et al. Valutazioni della pressione arteriosa e del tono adrenergico durante soggiorno montano di bassa quota. G Ital Cardiol 1989; 19: 456-461.

4. $\quad$ Stenberg J, Ekblom B, Messin R. Hemodynamic response to work at simulated altitude, 4000m. J Appl Physiol 1964; 19: 431-440.

5. D'Este D, Mantovan R, Martino A, et al. Blood pressare changes at rest and durino affort in normotensive and hypartensive subjects in response to altitude acure hypoxia. G Ital Cardiol 1991; 21: 643-649.
6. Palatini P, Guzzardi G, Penzo M, et al. Effect of low and high altitude exposure on the blood pressure response to exercise. Cardiologia 1991; 36: 853-859.

7. Ponchia A, Biasin R, Tempesta T, Tiene M. Aspetti medici della popolazione dei frequentatori della montagna, frequenza di mal di montagna e di incidenti nella pratica alpinistica. In: Atti del Convegno " $10^{\circ}$ Corso di aggiornamento per medici di trekking e spedizioni extraeuropee". Passo del Pordoi, 14-15.06.2203. Ed. CAI, pag 22. 